\title{
Terceiro Setor na Saúde: a produção científica dos Congressos Brasileiros de Ciências Sociais e Humanas em Saúde na ABRASCO
}

Third Sector in Health: the scientific production of the Brazilian Congresses of Social Sciences and Humanities in Health in ABRASCO

Tercer Sector en la Salud: la producción científica de los Congresos Brasileños de Ciencias

Sociales y Humanas en Salud en ABRASCO

Renato Barboza ${ }^{1}$; Áurea Maria Zöllner Ianni ${ }^{2}$; Olga Sofia Fabergé Alves ${ }^{3}$; Ane Talita da Silva Rocha $^{4}$; Sabrina Daniela Lopes Viana ${ }^{5}$

\section{Resumo}

Analisou-se a produção científica referente ao tema emergente "Terceiro Setor" nos Congressos Brasileiros de Ciências Sociais e Humanas em Saúde, promovidos pela Associação Brasileira de Pós-Graduação em Saúde Coletiva (Abrasco) entre 1995 e 2011 e a inserção no campo da Saúde Coletiva. Realizou-se um estudo exploratório, descritivo, retrospectivo de abordagem quantitativa, baseado nos Anais dos cinco Congressos Brasileiros de Ciências Sociais e Humanas em Saúde, realizados pela Abrasco. A análise das evidências demonstrou a relevância da produção científica sobre o Terceiro Setor na Saúde, indicando que o tema ainda não atingiu o seu ponto de saturação. No lócus da Atenção Básica, o esquadrinhamento dos objetos investigados contribuirá para a análise das políticas públicas de saúde, bem como para o aperfeiçoamento da regulação do mix público-privado, com vistas ao fortalecimento da gestão do Sistema Único de Saúde e das práticas de saúde sob a perspectiva da determinação social do processo saúde-doença.

Descritores: Saúde Coletiva, Ciências Sociais e Saúde, Setor Privado, Atenção Primária à Saúde, Sistema Único de Saúde.

\section{Abstract}

We analyzed the scientific literature about the emerging theme "Third Sector" in the Brazilian Congresses of Social Sciences and Humanities in Health, promoted by the Brazilian Association of Graduate Studies in Public Health (Abrasco) between 1995 and 2011 and its inclusion in the Collective Health field. We performed an exploratory, descriptive, retrospective quantitative approach, based on the Annals of the five Brazilian Congresses of Social Sciences and Humanities in Health, conducted by Abrasco. The analysis of the evidences showed the relevance of scientific literature on the Third Sector in Health, indicating that the subject has not reached its saturation point. In the locus of primary care, we conducted the full exploration about the investigated objects,

${ }^{1}$ Mestre em Saúde Coletiva pela Coordenadoria de Controle de Doenças da Secretaria de Estado de Saúde de São Paulo (SES/SP). Pesquisador Científico V do Instituto de Saúde (IS) da Secretaria de Estado de Saúde de São Paulo (SES/SP). Rua Santo Antônio, 590, Bela Vista, São Paulo - SP. CEP: 01314-000. email: renbarprof@ gmail.com

${ }^{2}$ Livre-Docente em Ciências Sociais em Saúde pela Universidade de São Paulo (USP). Professora Associada do Programa de Pós-graduação em Saúde Pública. Faculdade de Saúde Pública da Universidade de São Paulo (FSP-USP). Av. Dr. Arnaldo, 715, Pinheiros, São Paulo-SP, Brasil. CEP: 01246-904. email: aureanni@usp.br

${ }^{3}$ Mestre em História da Ciência pela Universidade de São Paulo (USP). Pesquisadora Científica IV do Instituto Butantan da Secretaria de Estado da Saúde de São Paulo (SES/SP). email: olga.alves@ @utantan.gov.br

${ }^{4}$ Mestre em Antropologia pela Faculdade de Filosofia Letras e Ciências Humanas da Universidade de São Paulo (FFLCH-USP). Curadora Cultural do Serviço Social do Comércio (SESC) São Paulo. email: ane.rocha@usp.br

${ }^{5}$ Mestre em Saúde Pública pela Faculdade de Saúde Pública da Universidade de São Paulo (FSP-USP). Professora do Centro Universitário Adventista de São Paulo. email: sabrinadlv@yahoo.com.br
} 
ISSN 2179-6750

which contribute to the analysis of public health policies, as well as to improve the regulation of the public-private mix, in order to strengthen the management of the National Health System and health practices from the perspective of social determination of the health-disease.

Key-words: Collective Health, Social and Health Sciences, Private Sector, Primary Health Care, Unified Health System

\section{Resumen}

Se analizó la literatura científica sobre el tema emergente "tercer sector" en los Congresos Brasileños de Ciencias Sociales y Humanas en Salud, promovido por la Asociación Brasileña de Estudios de Posgrado en Salud Pública (ABRASCO) entre 1995 y 2011 y la inclusión en el ámbito de la salud colectiva. Se realizó un estudio exploratorio, descriptivo y retrospectivo enfoque cuantitativo, basado en los Anales de los cinco Congresos Brasileños de Ciencias Sociales y Humanas en Salud, realizado por ABRASCO. El análisis demostró la importancia de la literatura científica sobre el tercer sector en la salud, lo que indica que el tema aún no ha alcanzado su punto de saturación. En el lugar de la atención primaria, el minucioso examen de los objetos investigados contribuir al análisis de las políticas de salud pública y para mejorar la regulación de la mezcla público-privada, con el fin de fortalecer la gestión del sistema de salud y las prácticas de salud desde la perspectiva de la determinación social de la salud-enfermedad.

Palabras-claves: Salud Colectiva, Ciencias Sociales y Salud, Sector Privado, Atención Primaria de Salud, Sistema Único de Salud.

\section{Introdução}

No Brasil os estudos referentes à área das Ciências Sociais em Saúde são recentes e remontam aos anos 70. Essas pesquisas foram impulsionadas após a criação da Associação Brasileira de Pós-Graduação em Saúde Coletiva (Abrasco) em 1979, sendo um marco na institucionalização não apenas do campo da Saúde Coletiva como também do subcampo das Ciências Sociais em Saúde no país ${ }^{1,2,3}$.

Ao investigar a produção científica nas Escolas de Ciências Sociais, Marsiglia et al ${ }^{4}$, verificaram que $17 \%$ dos objetos referem-se a temas "emergentes" no campo da saúde, destacandose na Pós-Graduação, temas relativos à saúde e trabalho e à qualidade de vida e ambiente, incrementados a partir da década de 1990.

Dentre os temas que fazem parte da trajetória das Ciências Sociais em Saúde no Brasil e ancorados na perspectiva teórica de Marsiglia et al ${ }^{4}$, elegemos a discussão sobre o Terceiro Setor no campo da Saúde Coletiva, por constituir-se um tema emergente, contínuo e ainda presente na gestão da política de saúde no âmbito do Sistema Único de Saúde (SUS). Essa política, promulgada pela Constituição Federal de 1988 reconheceu a saúde como um direito constitucional e foi implementada e institucionalizada por meio das Leis 8.080 e 8.142, ambas de 1990, em vigência no país ${ }^{5,6}$. Na saúde, o chamado Terceiro Setor compreende um conjunto de organizações privadas, 
ISSN 2179-6750

com finalidades e interesses públicos, as quais atuam de forma paralela e complementar ao Estado e ao Mercado, denominados respectivamente, Primeiro e Segundo Setor.

Em diversos países, as organizações circunscritas ao Terceiro Setor possuem segundo Salamon ${ }^{7}$, um conjunto de atributos comuns que permitem o seu reconhecimento, a saber: a) apresentam alguma forma de organização institucional legal ou não; b) são privadas e não vinculadas a estruturas governamentais; c) funcionam com sistemas de autogestão; d) não possuem fins lucrativos e os excedentes são investidos no aperfeiçoamento da própria organização; e e) desempenham em algum grau o trabalho voluntário.

No Brasil, o crescimento do Terceiro Setor pode ser situado em perspectiva histórica, como um dos desfechos da implementação do Plano Diretor da Reforma do Aparelho do Estado, conduzido a partir de 1995 pelo Ministro do Planejamento, Luiz Carlos Bresser Pereira durante o primeiro mandato do presidente Fernando Henrique Cardoso ${ }^{8}$. Essa reforma induziu e institucionalizou uma racionalidade baseada na redefinição do papel do Estado brasileiro na gestão pública, sobretudo na relação com as organizações da sociedade civil organizada. Esse novo marco legal permitiu a transferência das funções executivas do Estado para o Terceiro Setor, por meio de parcerias e mecanismos de financiamento, aperfeiçoados nos governos subsequentes e que estão em discussão no período atual, com vistas à publicização da gestão dos recursos governamentais ${ }^{8}$.

Constata-se o crescimento desde a década de 1990 no número de organizações do Terceiro Setor, dedicadas à prestação e à gerência de serviços em várias áreas sociais, nas três esferas de gestão da administração pública, sobretudo na área da saúde. Em 2010, segundo dados oficiais, existiam no país 290,7 mil fundações privadas e associações sem fins lucrativos, representando $5,2 \%$ das entidades públicas e privadas, lucrativas ou não, vinculadas ao Cadastro Central de Empresas do Instituto Brasileiro de Geografia e Estatística (IBGE). Entre 2006 e 2010, houve um incremento de $8,8 \%$ dessas entidades, contudo menor do que o verificado no período de 2002 a 2005 que atingiu 22,6\%. Segundo o levantamento, 18,6\% atuavam na implementação de políticas públicas nas áreas da saúde, educação, pesquisa e assistência social. No país, o contingente de pessoal remunerado na área da saúde foi o que mais empregou em 2010, perfazendo 574,5 mil trabalhadores 9 .

No que tange à proposição e à condução das ações, o Terceiro Setor destaca-se pela complexidade e heterogeneidade das organizações que o compõe, materializado em concepções e racionalidades distintas. Ao problematizar o desenvolvimento desse Setor, Gohn ${ }^{10}$ afirma que: 
múltiplas facetas. É contraditório, pois inclui tanto entidades progressistas como conservadoras. Abrange programas e projetos sociais que objetivam tanto a emancipação dos setores populares e a construção de uma sociedade mais justa, igualitária, com justiça social, como programas meramente assistenciais, compensatórios, estruturados segundo ações estratégico-racionais, pautadas pela lógica de mercado. Um ponto em comum: todos falam em nome da cidadania". (Gohn, 2000, p. 60)

Na trajetória da Reforma Sanitária Brasileira, a VIII Conferência Nacional de Saúde, realizada em Brasília em 1986, foi um marco na definição das bases conceituais que sustentaram a implantação do SUS. Esse movimento congregou profissionais da saúde, intelectuais, parlamentares e militantes de diversos movimentos sociais atuantes no processo de redemocratização do país, resultando em debates que configuraram o campo da Saúde Coletiva no Brasil ${ }^{11}$. Na conceituação de Jairnilson Paim ${ }^{12}$, militante e pesquisador do projeto da reforma sanitária, o campo da Saúde Coletiva compreende:

\footnotetext{
"simultaneamente, um campo científico e um âmbito de práticas, contribuindo com a Reforma Sanitária Brasileira mediante produção de conhecimentos e sua socialização junto aos movimentos sociais. Como campo científico, distancia-se da saúde pública institucionalizada e, como práxis, tem a possibilidade de radicalizar seus compromissos históricos com o povo, com as pessoas, com as classes dominadas.” (Paim, 2007, p. 2522)
}

Assim, aperfeiçoar as relações e interfaces entre o poder público e a sociedade civil, principalmente nos municípios, lócus privilegiado para a concretização das políticas, das ações e das práticas de saúde, pode contribuir para a democratização do Estado e para uma maior consciência política das instituições que atuam no Terceiro Setor ${ }^{13}$. Nessa ótica, a participação popular e o controle social da política de saúde, por meio do fortalecimento das conferências e dos conselhos de saúde, sobretudo os conselhos gestores das unidades de saúde, ainda incipientes em nosso país, representam alguns dos desafios a serem superados para assegurar a transparência da gestão com vistas à consolidação dos princípios da universalidade e da integralidade preconizados pelo SUS 5 .

A mobilização permanente da sociedade civil e a repolitização do SUS na perspectiva da reforma sanitária são elementos fundamentais que integram o Pacto pela Saúde, publicado em 2006 em vigência, o qual contempla os Pactos pela Vida, em Defesa do SUS e de Gestão e define um conjunto de responsabilidades sanitárias a partir de prioridades pactuadas pelos gestores para melhoria da saúde da população brasileira, bem como a promoção de inovações nos processos e nos 
ISSN 2179-6750

instrumentos de gestão, com vistas à maior eficiência e qualidade das ações de saúde ${ }^{14}$.

Nesse cenário, cabe frisar a relevância dos estudos sobre o Terceiro Setor na Saúde, quanto à concepção adotada, aos arranjos e práticas institucionais e, sobretudo os impactos na gestão no SUS para o seu aperfeiçoamento e consolidação na atualidade. $O$ presente artigo tem por objetivo analisar a produção científica referente ao tema Terceiro Setor nos Congressos Brasileiros de Ciências Sociais e Humanas em Saúde, promovidos pela Abrasco entre 1995 e 2007 e a inserção no campo da Saúde Coletiva.

\section{Metodologia}

Os dados apresentados no presente artigo são um desdobramento do projeto de pesquisa "Questões Contemporâneas nas Ciências Sociais em Saúde: o estudo de temas emergentes nos Congressos Brasileiros de Ciências Sociais e Humanas em Saúde, Abrasco 1995 - 2011”, financiado pelo $\mathrm{CNPq}^{15}$.

Entre 1995 a 2011 a Abrasco realizou cinco Congressos Brasileiros de Ciências Sociais e Humanas em Saúde, no intuito de discutir temas e propor uma agenda para o desenvolvimento desse subcampo, vinculado à Saúde Coletiva. Esses Congressos expressam em última instância a trajetória de institucionalização do subcampo das Ciências Sociais em Saúde, posteriormente denominado de Ciências Sociais e Humanas em Saúde, podendo ser considerado um dos principais lócus para socialização e problematização do conjunto da produção e da dinâmica desse subcampo 16

A presente pesquisa foi delineada como um estudo retrospectivo, exploratório-descritivo de abordagem quantitativa, cuja fonte de coleta dos dados foi baseada nos Livros de Resumos dos cinco Congressos Brasileiros de Ciências Sociais e Humanas em Saúde, realizados pela Abrasco. Conduziu-se a identificação e a sistematização dos trabalhos científicos sobre o Terceiro Setor na Saúde, objeto do presente estudo, selecionados pelas comissões científicas, apresentados e publicados nos Anais dos respectivos congressos. Apesar das limitações desse tipo de fonte documental, sobretudo a restrição do número de palavras, foi possível identificar e analisar a relevância desse tema na perspectiva da sua evolução no subcampo das Ciências Sociais e Humanas em Saúde.

No que se refere ao I e ao II Congressos, que não dispunham de CD dos Anais, a seleção dos trabalhos foi feita por meio da leitura de cada um dos trabalhos publicados tendo por referência as palavras-chave que cobriram o escopo do tema e orientaram a seleção dos resumos. Para o III, IV e V Congressos, a seleção foi feita por busca ativa das palavras-chave nos CDs e nos sites específicos 
ISSN 2179-6750

dos congressos. Nos cinco congressos foram aferidos 7.517 resumos que compõem o universo de análise da presente pesquisa. Para a seleção dos trabalhos utilizaram-se as palavras-chave: participação; movimentos sociais; participação social; sociedade civil; Terceiro Setor; ONG (Organização Não Governamental); controle social; voluntariado; rede social solidária; accountability social; rede de cooperação; rede de interesse; organizações sociais; organizações sociais da saúde; intersetorialidade; sustentabilidade e terceirização.

Os dados coletados foram compilados em um banco de dados contendo o registro das informações de cada resumo selecionado, o que permitiu sua categorização e classificação segundo diferentes perspectivas. $\mathrm{O}$ banco foi construído com variáveis intencionalmente definidas pelos pesquisadores, a saber: congresso, vínculo institucional dos autores (Academia, Serviço de Saúde/Secretaria ou ONG), estado de origem da instituição, macrorregião, tipo de instituição acadêmica (Federal, Estadual, Privada), tipo de estudo (pesquisa ou relato de experiência), metodologia, modalidade de apresentação (oral, pôster, pôster eletrônico ou publicação nos Anais) e objetos identificados na Atenção Básica. Os dados foram organizados em um banco de dados em planilha Excel e submetidos à análise descritiva baseada em frequências absolutas e relativas.

O projeto foi aprovado pelo Comitê de Ética em Pesquisa do Instituto de Saúde da Secretaria de Estado da Saúde de São Paulo (protocolo nº 013/2010).

\section{Resultados e Discussão}

No universo dos 7.517 trabalhos apresentados nas cinco edições dos Congressos Brasileiros de Ciências Sociais e Humanas em Saúde foram encontrados 126 trabalhos referentes ao tema Terceiro Setor, perfazendo $1,7 \%$ do total analisado. No período entre o I e o IV congressos da Abrasco, verificou-se um incremento de oito vezes no total dos trabalhos apresentados pelas instituições, com redução no percentual aferido na última edição. A participação dos resumos do Terceiro Setor ocorreu em todos os congressos analisados entre 1995 e 2011. A proporção de trabalhos desse tema foi crescente a cada congresso, variando de $3,2 \%$ a $69,8 \%$. Chama a atenção, a concentração de trabalhos sobre o tema nas últimas duas edições, superior a $85 \%$, indicando um crescimento de quatro vezes na produção nesse período. (Tabela 1)

Ao analisarmos os livros de resumos publicados pela Abrasco, constatamos que entre as temáticas definidas pela comissão científica, as relacionadas ao Terceiro Setor, para submissão de trabalhos, abordaram no I Congresso, os movimentos sociais, a participação em saúde e os conselhos de saúde. No II Congresso, os temas foram mais amplos e abordaram a reforma do Estado e a Saúde, as novas formas de gestão, o controle social, a promoção da saúde e a 
ISSN 2179-6750

intersetorialidade. O III Congresso foi estruturado em vários eixos temáticos com subtemas, revelando a complexidade da produção das Ciências Sociais em Saúde. Nesse Congresso houve um eixo dedicado à sociedade civil, movimentos sociais e cultura, subdivididos em temas, como a relação público/privado na saúde, a participação social e democracia, as redes de apoio social, as estratégias e práticas em saúde desenvolvidas pela sociedade civil junto a grupos específicos. No IV Congresso essa estrutura foi mantida, destacando-se um eixo temático sobre mobilização e participação social na Saúde Coletiva, com vários subtemas tais como: a participação e o controle social, as demandas sociais e o direito à saúde, a mobilização da sociedade civil e os movimentos sociais na defesa da saúde, os movimentos identitários, novos modelos de gestão e organização dos serviços de saúde. Por fim, no V Congresso houve uma mudança no formato e os trabalhos foram classificados e submetidos segundo grupos temáticos, com o fito de aprofundar o debate e o intercâmbio da produção científica. Nesse Congresso um dos grupos temáticos contemplou os debates sobre os movimentos sociais e a educação popular em saúde.

Tabela 1. Frequência absoluta e relativa dos trabalhos científicos e do Terceiro Setor apresentados nos I, II, III, IV e V Congressos Brasileiros de Ciências Sociais e Humanas em Saúde da Abrasco, 1995 - 2011.

\begin{tabular}{ccccc}
\hline Congresso & \multicolumn{2}{c}{ Trabalhos } & \multicolumn{2}{c}{ Terceiro Setor } \\
& $\mathbf{N}$ & $\mathbf{\%}$ & $\mathbf{N}$ & $\mathbf{\%}$ \\
\hline I & 398 & 5,3 & 4 & 3,2 \\
II & 437 & 5,8 & 2 & 1,6 \\
III & 2032 & 27,0 & 11 & 8,7 \\
IV & 3168 & 42,1 & 21 & 16,7 \\
V & 1482 & 19,7 & 88 & 69,8 \\
Total & $\mathbf{7 5 1 7}$ & $\mathbf{1 0 0}$ & $\mathbf{1 2 6}$ & $\mathbf{1 0 0}$ \\
\hline \multicolumn{7}{l}{}
\end{tabular}

$\mathrm{Na}$ análise da variável vínculo institucional do autor principal, constatou-se que os trabalhos, majoritariamente são oriundos de instituições acadêmicas, presentes em todos os congressos e responderam por $64,2 \%$ dos resumos sobre o Terceiro Setor. Em segundo lugar, nesse ranque, 9,5\% foram apresentados por instituições de saúde, caracterizadas como serviços, incluindo unidades de saúde e secretarias das três esferas de gestão do SUS. Por fim, os autores vinculados à modalidade ONG, a qual inclui associações, fundações e diversas organizações tipificadas no Terceiro Setor, registraram-se apenas 7,9\% dos trabalhos. Cabe ponderar que o perfil acadêmico predomina nos Congressos de Ciências Sociais em Saúde, no entanto constatou-se a participação crescente dos serviços de saúde e em menor grau das ONG. Todos esses atores compõe o campo da Saúde 
ISSN 2179-6750

Coletiva e também transitam no subcampo em questão. (Tabela 2)

Chama a atenção que a participação dos trabalhos sobre o Terceiro Setor, nas modalidades, acadêmico e serviços foi crescente desde o III Congresso, sendo que os primeiros quintuplicaram a sua participação, comparada ao último Congresso. Quanto aos serviços, apesar de apresentarem um número reduzido de resumos comparado aos acadêmicos, octuplicaram a sua produção. No que se refere às ONGs, a produção foi um pouco menor do que a dos serviços, verificando-se uma concentração de resumos no V Congresso. (Tabela 2)

Tabela 2. Frequência absoluta e relativa dos trabalhos científicos sobre o tema Terceiro Setor apresentados nos Congressos Brasileiros de Ciências Sociais e Humanas em Saúde da Abrasco, segundo vínculo institucional dos autores e parcerias, $1995-2011$.

\begin{tabular}{ccccccccc}
\hline Congresso & \multicolumn{2}{c}{ Acadêmico } & \multicolumn{2}{c}{ Serviços } & \multicolumn{2}{c}{ ONG } & \multicolumn{2}{c}{ Parcerias } \\
\cline { 2 - 9 } & $\mathbf{N}$ & $\mathbf{\%}$ & $\mathbf{N}$ & $\mathbf{\%}$ & $\mathbf{N}$ & $\mathbf{\%}$ & $\mathbf{N}$ & $\mathbf{\%}$ \\
I & 3 & 3,7 & 0 & 0,0 & 1 & 10,0 & 0 & 0,0 \\
II & 1 & 1,2 & 0 & 0,0 & 1 & 10,0 & 0 & 0,0 \\
III & 10 & 12,3 & 1 & 8,3 & 0 & 0,0 & 0 & 0,0 \\
IV & 17 & 21,0 & 3 & 25,0 & 1 & 10,0 & 0 & 0,0 \\
V & 50 & 61,7 & 8 & 66,7 & 7 & 70,0 & 23 & 100 \\
Total & $\mathbf{8 1}$ & $\mathbf{1 0 0}$ & $\mathbf{1 2}$ & $\mathbf{1 0}$ & $\mathbf{1 0}$ & $\mathbf{1 0 0}$ & $\mathbf{2 3}$ & $\mathbf{1 0 0}$ \\
\hline
\end{tabular}

Os dados evidenciaram a importância dos trabalhos desenvolvidos em parceria entre esses três tipos de instituições, alcançando 18,2\% (23 trabalhos) do total dos resumos analisados. Todos os trabalhos implementados em interface, concentraram-se no último Congresso, revelando uma nova modalidade no desenvolvimento de projetos de pesquisa ou de relatos de experiência, a qual merece ser monitorada no futuro para verificarmos qual será a tendência. Nesse cenário, as instituições acadêmicas foram as que mais estabeleceram parcerias, sobretudo com os serviços e as ONG. (Tabela 2)

É possível que essas alianças interinstitucionais, ainda emergentes, possam revelar um movimento crescente no campo da Saúde Coletiva nos últimos anos, no qual se observa uma maior interação entre as instituições públicas e as organizações que atuam no Terceiro Setor na saúde, resultado do processo da reforma do Estado brasileiro nos anos $1990^{8}$.

Nos trabalhos científicos apresentados pelas universidades públicas, incluindo as que estão sob gestão estadual ou federal e pelas universidades privadas, verificou-se nessa tipologia, o predomínio de trabalhos conduzidos pelas universidades federais, perfazendo 62,7\% do total. Esse 
ISSN 2179-6750

cenário está em consonância com a maior participação de pesquisadores oriundos da academia nos Congressos estudados e também reflete a estrutura e a oferta concentrada de cursos e vagas no país nas universidades federais ${ }^{17}$. Na sequência, observamos a participação das universidades estaduais, correspondendo a $22,3 \%$ dos trabalhos e por último, as universidades privadas, com apenas $8 \%$ dos resumos. (Tabela 3)

Aferiu-se um aumento considerável na proporção dos trabalhos das universidades federais e estaduais entre o III e o V Congressos, observando-se nesse período um incremento na produção de $72,6 \%$ e 45,5\%, respectivamente. Entre as universidades privadas também houve aumento, corroborando para a importância dos estudos sobre o Terceiro Setor na academia. Constatou-se ainda que $7 \%$ dos trabalhos foram desenvolvidos em parceria entre as instituições acadêmicas, sobretudo nas universidades estaduais e federais, concentrada na última edição, indicando assim, a relevância dessas alianças na condução da produção de conhecimento no subcampo das Ciências Sociais e Humanas em Saúde. (Tabela 3)

Tabela 3. Frequência absoluta e relativa dos trabalhos científicos sobre o tema Terceiro Setor apresentados nos Congressos Brasileiros de Ciências Sociais e Humanas em Saúde da Abrasco, segundo o tipo de instituição acadêmica, $1995-2011$.

\begin{tabular}{ccccccccc}
\hline Congresso & \multicolumn{2}{c}{ Estadual } & \multicolumn{2}{c}{ Federal } & \multicolumn{2}{c}{ Privada } & \multicolumn{2}{c}{ Parcerias } \\
\cline { 2 - 9 } & $\mathbf{N}$ & $\mathbf{\%}$ & $\mathbf{N}$ & $\mathbf{\%}$ & $\mathbf{N}$ & $\mathbf{\%}$ & $\mathbf{N}$ & $\mathbf{\%}$ \\
I & 0 & 0,0 & 3 & 4,8 & 0 & 0,0 & 0 & 0,0 \\
II & 1 & 4,5 & 0 & 0,0 & 0 & 0,0 & 0 & 0,0 \\
III & 3 & 13,6 & 2 & 3,2 & 3 & 37,5 & 2 & 28,6 \\
IV & 5 & 22,7 & 10 & 16,1 & 0 & 0,0 & 0 & 0,0 \\
V & 13 & 59,1 & 47 & 75,8 & 5 & 62,5 & 5 & 71,4 \\
Total & $\mathbf{2 2}$ & $\mathbf{1 0 0}$ & $\mathbf{6 2}$ & $\mathbf{1 0 0}$ & $\mathbf{8}$ & $\mathbf{1 0 0}$ & $\mathbf{7}$ & $\mathbf{1 0 0}$ \\
\hline \multicolumn{4}{c}{ Fonte: Anais dos Congressos Abrasco. }
\end{tabular}

Os trabalhos sobre o Terceiro Setor foram oriundos da maioria das macrorregiões brasileiras, exceto a região Norte, onde não foi encontrado nenhum resumo. Constatou-se que houve concentração de trabalhos provenientes das instituições localizadas nas regiões Sudeste e Nordeste, perfazendo, respectivamente, $52,6 \%$ e $27,1 \%$ dos resumos analisados, contribuindo com quase $80 \%$ da produção nacional sobre o tema em questão. Nas demais regiões, verificaram-se instituições oriundas do Sul $(13,1 \%)$ e em menor proporção do Centro-Oeste com $7 \%$ dos resumos. (Tabela 4) Cabe destacar que essa tendência de concentração de trabalhos na região Sudeste, tem sido observada em outros Congressos promovidos pela Abrasco, seja no Congresso de Brasileiro de 
ISSN 2179-6750

Epidemiologia, no Congresso de Política, Planejamento e Gestão, ou ainda no "Abrascão", realizado a cada três anos. Corroborando esse cenário marcado pela reprodução das desigualdades do capital intelectual brasileiro, vale destacar que a maioria dos pesquisadores que atuam no campo da Saúde Coletiva estão lotados em instituições acadêmicas do eixo Sul-Sudeste ${ }^{3}$.

Tabela 4. Frequência absoluta e relativa dos trabalhos científicos sobre o tema Terceiro Setor apresentados nos Congressos Brasileiros de Ciências Sociais e Humanas em Saúde da ABRASCO, segundo a região de origem da instituição, 1995 - 2011.

\begin{tabular}{ccccccccccc}
\hline Congresso & \multicolumn{2}{c}{$\mathbf{C O}$} & \multicolumn{2}{c}{$\mathbf{N E}$} & \multicolumn{2}{c}{$\mathbf{N}$} & \multicolumn{3}{c}{ SE } & \multicolumn{2}{c}{ S } \\
\cline { 2 - 12 } & $\mathbf{N}$ & $\mathbf{\%}$ & $\mathbf{N}$ & $\mathbf{\%}$ & $\mathbf{N}$ & $\mathbf{\%}$ & $\mathbf{N}$ & $\mathbf{\%}$ & $\mathbf{N}$ & $\mathbf{\%}$ \\
I & 0 & 0,0 & 2 & 6,5 & 0 & 0,0 & 2 & 3,3 & 0 & 0,0 \\
II & 0 & 0,0 & 0 & 0,0 & 0 & 0,0 & 1 & 1,7 & 0 & 0,0 \\
III & 1 & 12,5 & 2 & 6,5 & 0 & 0,0 & 4 & 6,7 & 4 & 26,7 \\
IV & 1 & 12,5 & 4 & 12,9 & 0 & 0,0 & 10 & 16,7 & 2 & 13,3 \\
V & 6 & 75,0 & 23 & 74,2 & 0 & 0,0 & 43 & 71,7 & 9 & 60,0 \\
Total & $\mathbf{8}$ & $\mathbf{1 0 0}$ & $\mathbf{3 1}$ & $\mathbf{1 0 0}$ & $\mathbf{0}$ & $\mathbf{0 , 0}$ & $\mathbf{6 0}$ & $\mathbf{1 0 0}$ & $\mathbf{1 5}$ & $\mathbf{1 0 0}$ \\
\hline
\end{tabular}

Fonte: Anais dos Congressos Abrasco.

Analisando-se, especificamente, a produção científica das regiões Sudeste e Nordeste, constatou-se que entre o III e o V Congressos, ambas tiveram um incremento dos resumos apresentados. (Tabela 4) Quanto à distribuição, segundo o vínculo do autor principal por Unidade da Federação, 17 estados, além do Distrito Federal apresentaram resumos sobre a temática no período entre 1995 e 2011. Rio de Janeiro e São Paulo corresponderam a 91,7\% do total dos resumos apresentados na região Sudeste e quase a metade da produção nacional, alcançando 48,2\%. Na região Nordeste, Bahia, Ceará, Alagoas e Pernambuco responderam por $83,8 \%$ da produção regional e $22,8 \%$ do país. Como mencionado anteriormente, esse fato pode estar associado a uma concentração das universidades e dos institutos de pesquisa que atuam no campo da Saúde Coletiva, sobretudo, no eixo Rio de Janeiro - São Paulo - Bahia. Apesar da menor participação das regiões, Centro-Oeste e Sul, também houve aumento da produção registrada nos últimos dois Congressos. Em grande angular, pode-se afirmar que o tema Terceiro Setor ainda não atingiu o seu ponto de saturação, observando-se nas duas últimas edições desses Congressos uma tendência de crescimento no total de trabalhos na maioria das macrorregiões brasileiras.

Aferiram-se, ainda sete trabalhos apresentados no V Congresso, conduzidos por instituições vinculadas a mais de uma macrorregião, o que talvez possa indicar uma tendência futura, a depender da evolução da produção, um processo multicêntrico de articulação das instituições 
ISSN 2179-6750

interessadas na investigação do tema do Terceiro Setor. Observou-se, também, a participação de instituições de outros países com a submissão de quatro resumos (Costa Rica, Espanha e Estados Unidos). Em que pese o número reduzido desses trabalhos, esse fato evidencia um processo de internacionalização empreendido pela Abrasco nos últimos anos. Porém, como todos esses resumos estão concentrados no III Congresso, resta saber se esse movimento se constituirá em uma tendência à internacionalização.

Quanto ao tipo de estudo, os trabalhos foram classificados em duas variáveis: resumo de pesquisa e relato de experiência. No conjunto dos cinco Congressos, predominaram os resumos de pesquisa, $72,2 \%$, contra 27,8\% dos relatos de experiência. Desde o III Congresso, os resumos de pesquisa apresentaram uma tendência de crescimento, tendo quintuplicado sua produção até a última edição, evidenciando o interesse das diversas instituições de pesquisa pelo tema do Terceiro Setor. A modalidade dos relatos de experiência, mesmo em menor proporção, quadruplicou a sua produção nas duas últimas edições, sobretudo no V Congresso. Pode-se afirmar que esse tema emergente ainda carece de investigações que contribuam para o seu esquadrinhamento em nossa sociedade e requer maior elucidação da dinâmica estabelecida por meio das interfaces crescentes, desde os anos 1990, com os demais setores: o Estado e o Mercado.

No que concerne à análise da metodologia adotada (Tabela 5), verificou-se que $80,1 \%$ dos resumos classificados como pesquisas ou relatos de experiências foram ancorados na abordagem qualitativa, o que está em consonância com o perfil dos Congressos de Ciências Sociais em Saúde da Abrasco e com a tendência de incorporação desses métodos nos estudos realizados na última década no campo da Saúde Coletiva ${ }^{18}$. A segunda abordagem metodológica mais referida foi a quantitativa, perfazendo $11,1 \%$ dos trabalhos, seguida das abordagens mistas com 8,0\%. As metodologias quanti-qualitativas apareceram nos estudos, a partir do III Congresso, mantendo-se até o V Congresso, o que pode indicar uma tendência de diversificação das metodologias adotadas pelos pesquisadores no subcampo das Ciências Sociais e Humanas em Saúde para explicar a complexidade crescente dos objetos investigados na perspectiva interdisciplinar da Saúde Coletiva 17 .

Quanto à modalidade de apresentação nos Congressos, 41,3\% dos trabalhos sobre o Terceiro Setor foram apresentados na modalidade oral, crescente desde o III Congresso e ampliada em onze vezes na comparação com o último Congresso. Esse fato também evidencia a relevância e o destaque conferido ao tema pela Abrasco. A apresentação por meio de pôsteres correspondeu a 16,7\%. Cabe destacar, que na última edição foram introduzidas duas novas modalidades de apresentação: pôster eletrônico e publicação no Livro de Resumos, ambas responsáveis por $42 \%$ das 
ISSN 2179-6750

apresentações sobre o tema em questão e equilibradas proporcionalmente entre si, assim como em relação aos trabalhos apresentados na modalidade oral.

Tabela 5. Frequência absoluta e relativa dos trabalhos científicos sobre o tema Terceiro Setor apresentados nos Congressos Brasileiros de Ciências Sociais e Humanas em Saúde da Abrasco, segundo a metodologia, 1995 - 2011.

\begin{tabular}{ccccccccc}
\hline Congresso & \multicolumn{2}{c}{ Qualitativa } & \multicolumn{2}{c}{ Quantitativa } & \multicolumn{2}{c}{ Quanti/Quali } & \multicolumn{2}{c}{ Sem inf. } \\
\cline { 2 - 9 } & $\mathbf{N}$ & $\mathbf{\%}$ & $\mathbf{N}$ & $\mathbf{\%}$ & $\mathbf{N}$ & $\mathbf{\%}$ & $\mathbf{N}$ & $\mathbf{\%}$ \\
I & 4 & 4,0 & 0 & 0,0 & 0 & 0,0 & 0 & 0,0 \\
II & 2 & 2,0 & 0 & 0,0 & 0 & 0,0 & 0 & 0,0 \\
III & 8 & 7,9 & 1 & 7,1 & 2 & 20,0 & 0 & 0,0 \\
IV & 18 & 17,8 & 1 & 7,1 & 2 & 20,0 & 0 & 0,0 \\
V & 69 & 68,3 & 12 & 85,7 & 6 & 60,0 & 1 & 100 \\
Total & $\mathbf{1 0 1}$ & $\mathbf{1 0 0}$ & $\mathbf{1 4}$ & $\mathbf{1 0 0}$ & $\mathbf{1 0}$ & $\mathbf{1 0 0}$ & $\mathbf{1}$ & $\mathbf{1 0 0}$ \\
\hline \multicolumn{3}{c}{ Fonte: Anais dos Congressos Abrasco. }
\end{tabular}

No esquadrinhamento da produção científica sobre o Terceiro Setor na Saúde, encontramos 60 trabalhos conduzidos pelas instituições no lócus da Atenção Básica, perfazendo 47,6\% dos resumos analisados (Tabela 6). Majoritariamente esses trabalhos foram oriundos da Academia (61,7\%); seguido da modalidade de parcerias entre a Academia, os Serviços e as ONG (20\%); Serviços $(11,7 \%)$ e ONG (6,7\%). Observa-se no topo do ranque dos objetos investigados na Atenção Básica, que mais de um terço dos trabalhos referiu-se às ações desenvolvidas em rede ancoradas na diretriz intersetorial e na educação em saúde. Na contemporaneidade esses dois temas representam importantes desafios para consolidar a atenção primária à saúde e reorientar o modelo de atenção à população, como preconizado pela Política Nacional de Atenção Básica, consonante ao Pacto pela Saúde em vigência no país e aos princípios do SUS ${ }^{19,14,5,6}$. No que tange às práticas de saúde, destacaram-se a condução de processos baseados em ações meio, como as atividades de educação em saúde (20\%) e de promoção à saúde e prevenção primária de agravos (13,3\%), voltadas à população em geral, aos idosos, aos povos indígenas e ao enfrentamento de problemas, como o uso abusivo de álcool e outras drogas, a aids, a violência sexual contra crianças e adolescentes e a prevenção da anemia falciforme entre a população negra.

Dentre os cinco objetos principais identificados, elencamos na Tabela 6, os trabalhos que abordaram a atuação dos movimentos sociais e da participação na saúde $(13,3 \%)$ e as ações implementadas por meio de parcerias e interfaces entre as Secretarias de Saúde Estaduais e Municipais e as organizações da sociedade civil, tipificadas como ONG (8,3\%). O escopo dessas 
ISSN 2179-6750

parcerias foi relacionado ao desenvolvimento de ações voltadas à saúde indígena e de gays, à saúde e ambiente, à educação popular e à formação profissional de agentes comunitários no âmbito da Estratégia Saúde da Família. Contudo, a prestação de serviços, sobretudo na modalidade de parcerias com o poder público e o acesso crescente ao financiamento estatal pós Reforma do Estado $^{8}$, essencial para a sustentabilidade das entidades do Terceiro Setor, corroboraram para que a participação social e o controle público da política de saúde nos colegiados do SUS ficassem relegados a um segundo plano. Assim, concordamos com Paim ${ }^{20}$, ao apontar que essas entidades acabam vinculando-se mais à "via técnico-institucional" do que à "via sócio-comunitária", reproduzindo uma racionalidade hegemônica na relação entre o Estado e a sociedade civil, a qual vulnerabiliza o desempenho dos papéis assumidos por essas organizações no SUS.

Tabela 6. Frequência absoluta e relativa dos trabalhos científicos sobre o tema Terceiro Setor apresentados nos Congressos Brasileiros de Ciências Sociais e Humanas em Saúde da Abrasco, segundo objetos investigados na Atenção Básica, 1995 - 2011.

\begin{tabular}{lcc}
\hline Objetos investigados na Atenção Básica & N & \% \\
\hline Intersetorialidade e redes & 13 & 21,7 \\
Educação em saúde & 12 & 20,0 \\
Movimentos sociais e participação na saúde & 8 & 13,3 \\
\hline Ações de promoção da saúde e prevenção & 5 & 8,3 \\
Parceria entre governo e ONGs & 5 & 8,3 \\
Gerencia de serviços e terceirização de recursos humanos & 5 & 8,3 \\
Direito à saúde & 4 & 6,8 \\
Atuação de ONGs na Saúde & 3 & 5,0 \\
Avaliação de políticas ou ações programáticas & 3 & 5,0 \\
Controle social nos conselhos de saúde & 2 & 3,3 \\
Total & $\mathbf{6 0}$ & $\mathbf{1 0 0}$ \\
\hline \multicolumn{1}{c}{ Fonte: Anais dos Congressos Abrasco. } & &
\end{tabular}

Chama a atenção que apenas dois trabalhos discutiram a questão do controle social nos conselhos de saúde, o qual é um elemento inovador na política de saúde pós Constituição cidadã e vital para a consolidação do SUS e do projeto da Reforma Sanitária brasileira na atualidade ${ }^{11}$. Nessa perspectiva a concepção e os modelos teóricos vigentes dedicados a explicar a participação e a representação social na saúde e em outras políticas sociais tornaram-se obsoletos e não conseguem mais apreender a emergência e a disseminação de novas modalidades de representação na contemporaneidade, materializadas em atores, pautas e lócus plurais ${ }^{21}$. Não obstante aos avanços e as lacunas do exercício do controle social nos conselhos de saúde e aos desafios inerentes à sua concretização na realidade heterogênea dos municípios brasileiros, envidar esforços para a 
ISSN 2179-6750

concretização desses espaços decisórios, sobretudo nas unidades da rede de Atenção Básica por meio dos conselhos locais gestores, ainda é um desafio a ser alcançado ${ }^{11,22}$. Cabe ainda frisar que as análises sobre o controle social na saúde e sobre a atuação dos movimentos sociais, organizados ou não como ONG, reiteram a relevância da contribuição do subcampo das Ciências Sociais e Humanas em Saúde ${ }^{2}$.

No que tange aos modelos adotados pelas Secretarias para gestão do SUS, 8,3\% dos trabalhos abordaram a questão da gerência de serviços e o processo de terceirização dos recursos humanos, sobretudo nas unidades da rede de Atenção Básica (Tabela 6). Os resumos sobre gestão do Terceiro Setor na Saúde discutem, principalmente, a delegação e os resultados da gerência de serviços de saúde por meio de organizações pertencentes à administração indireta no SUS. A discussão sobre a organização da força de trabalho no nível da Atenção Básica tem sido um objeto de estudo abraçado pela Academia, sendo uma questão relevante para o aperfeiçoamento da gestão dos sistemas municipais de saúde ${ }^{23}$. Nos anos 2000, com o advento da expansão da Estratégia Saúde da Família no país, constatou-se o crescimento de contratações de profissionais de nível superior e técnico, sob a gerência de instituições do Terceiro Setor, tipificadas como Organizações da Sociedade Civil de Interesse Público (OSCIP), Organizações Sociais da Saúde (OSS), além das cooperativas, sobretudo as constituídas por médicos ${ }^{24,25}$. Contudo, como alertam Barboza e Morais ${ }^{26}$,

\footnotetext{
“esse tipo de arranjo institucional revela vulnerabilidades na provisão, condução e organização dos recursos humanos no âmbito do sistema local de saúde. Dependendo da gestão municipal, há precarização dos vínculos de trabalho, sem garantias de renovação dos contratos, de direitos trabalhistas, gerando baixa adesão e alta rotatividade dos profissionais.” (Barboza e Morais, 2014, p.38)
}

Essas vulnerabilidades impactam não só a organização dos serviços e o processo de trabalho das equipes, sobretudo as práticas de saúde, as quais dependem do acolhimento dos usuários e do fortalecimento dos vínculos para assegurar a longitudinalidade do cuidado e o desenvolvimento de uma atenção primária à saúde eficaz na coordenação das ações no lócus das redes de atenção à saúde no país ${ }^{27,28,22}$.

\section{Considerações Finais}

A análise das evidências do presente estudo demonstrou a relevância da trajetória da produção científica sobre o Terceiro Setor na Saúde, socializada no lócus dos Congressos Brasileiros de Ciências Sociais e Humanas em Saúde promovidos pela Abrasco. O capital 
ISSN 2179-6750

intelectual produzido sobre o tema, sobretudo pela Academia, pelas secretarias e serviços de saúde e pelas ONGs contribuem para a análise das políticas públicas de saúde em curso no país, bem como para a atualização da agenda de pesquisa no campo da Saúde Coletiva.

A produção crescente de trabalhos apresentados pelas instituições no período analisado vocaliza a complexidade de temas e objetos relacionados ao Terceiro Setor na Saúde e aponta que o tema ainda não atingiu o seu ponto de saturação. Revela uma produção científica concentrada nas instituições oriundas das regiões Sudeste e Nordeste do país e nos estados do Rio de Janeiro e São Paulo, responsáveis por quase metade da produção nacional, o que pode ser explicado pelo maior número de pesquisadores e instituições dedicadas à Saúde Coletiva e ao subcampo das Ciências Sociais e Humanas em Saúde.

No que concerne à produção das instituições sobre os objetos relacionados à Atenção Básica, constatou-se a relevância do número de trabalhos dedicados a explorar essa temática no âmbito do Terceiro Setor na Saúde. Cabe destacar que o esquadrinhamento desses objetos poderá contribuir para o aperfeiçoamento das análises das políticas públicas de saúde, bem como para com o desenvolvimento de processos de regulação, cada vez mais complexos, que aprofundem o conhecimento sobre a relação e o mix público-privado, com vistas ao fortalecimento da gestão do SUS e a organização social das práticas de saúde sob a perspectiva da determinação social do processo saúde-doença.

Assim, reafirmamos a relevância do subcampo das Ciências Sociais e Humanas em Saúde como base para reflexão teórica, iluminando as lacunas e os desafios para compreendermos em perspectiva histórica, o desenvolvimento do Terceiro Setor na Saúde, e a construção do pensamento social em saúde no país, orientado pelo escopo do campo da Saúde Coletiva nas bases epistemológicas e das práticas sociais em prol da emancipação e do direito constitucional à saúde. Por fim, recomendamos o desenvolvimento de outros estudos sobre a produção científica e o impacto do Terceiro Setor na Saúde no campo das Ciências Sociais e Humanas em Saúde, com o fito de aprofundar a produção teórica e subsidiar o aprimoramento das políticas e das práticas de saúde no SUS, concebidas e implementadas na interface entre as instituições governamentais e não governamentais brasileiras.

\section{Referências}

1. Nunes ED. A trajetória das ciências sociais em saúde na América Latina: revisão da produção científica. Rev Saúde Pública 2006; 40(Nº esp.):64-72.

2. Minayo MCS. A Produção de Conhecimentos na Interface entre as Ciências Sociais e 32 
ISSN 2179-6750

Humanas e a Saúde Coletiva. Saúde Soc 2013; 22(1):21-31.

3. Ianni AMZ, Spadacio C, Barboza R, Alves OSF, Viana SDL, Rocha ATS. Os Congressos Brasileiros de Ciências Sociais e Humanas em Saúde da Abrasco: um campo científico em disputa. Ciênc. saúde colet. 2015; 20(2):503-513.

4. Marsiglia RMG, Spinelli SP, Lopes MF, Silva TCP. Das ciências sociais em saúde: produção científica de pós-graduação em ciências sociais. Ciênc. saúde colet. 2003; 8(1):275-285.

5. Lei $\mathrm{n}^{\circ} 8.080$ de 19 de Setembro de 1990. Dispõe sobre as condições para a promoção, proteção e recuperação da saúde, a organização e o funcionamento dos serviços correspondentes e dá outras providências. Diário Oficial da União 1990; 19 set.

6. Lei $n^{\circ} 8.142$ de 28 de Dezembro de 1990. Dispõe sobre a participação da comunidade na gestão do Sistema Único de Saúde e sobre as transferências intergovernamentais de recursos financeiros na área da saúde e dá outras providências. Diário Oficial da União 1990; 28 dez.

7. Salamon L. Estratégias para fortalecimento do terceiro setor. In: Ioschpe EB, organizador. Terceiro Setor e Desenvolvimento Sustentado. Rio de Janeiro: Paz e Terra; São Paulo: Gife; 1997. p. 89-112.

8. Presidência da República. Plano Diretor da Reforma do Aparelho do Estado. Brasília: Câmara da Reforma do Estado; 1995.

9. Instituto Brasileiro de Geografia e Estatística (Ibge). As fundações privadas e associações sem fins lucrativos no Brasil 2010. Rio de Janeiro: Ibge; 2012.

10. Gohn MG. Mídia, terceiro setor e MST: impacto sobre o futuro das cidades e do campo. Petrópolis: Vozes; 2000.

11. Paim J, Travassos C, Almeida C, Bahia L, Macinko J. O sistema de saúde brasileiro: história, avanços e desafios. Lancet 2011; Saúde no Brasil 1, p. 11-31.

12. Paim JS. Saúde Coletiva como compromisso: a trajetória da Abrasco. Cad Saúde Pública $2007 ; 23(10): 2521-2522$

13. Barboza R. Relação Estado e Sociedade CiviI: as parcerias com Organizações Não Governamentais no campo da AIDS e o controle social no SUS. In: Paiva V, França Júnior I, Kalichman AO, organizadores. Vulnerabilidade e direitos humanos - prevenção e promoção da saúde: planejar, fazer, avaliar. Curitiba: Juruá; 2013. p. 213-248.

14. Brasil. Ministério da Saúde. Portaria GM/MS n. 399, de 22/02/2006. Divulga o Pacto pela Saúde 2006 e aprova as Diretrizes Operacionais do referido Pacto, Brasília-DF, 2006.

15. Ianni AMZ, Barboza R, Alves OSF, Rocha ATS, Viana SDL, Torres R. Questões contemporâneas nas Ciências Sociais em Saúde: o estudo de temas emergentes nos 
ISSN 2179-6750

congressos brasileiros de Ciências Sociais e Humanas em Saúde, ABRASCO 1995 - 2007. [relatório final de pesquisa]. São Paulo; 2012.

16. Canesqui AM. As Ciências Sociais e Humanas em Saúde na Associação Brasileira de Pósgraduação em Saúde Coletiva. Physis 2008; 18(2):215-250.

17. Nunes ED, Ferreto LE, Oliveira ALO, Nascimento JL, Barros NF, Castellanos MEP. O campo da Saúde Coletiva na perspectiva das disciplinas. Ciênc. saúde colet. 2010; 15(4):1917-1922.

18. Luz MT, Mattos RS. Dimensões qualitativas na produção científica, tecnológica e na inovação em Saúde Coletiva. Ciênc. saúde colet. 2010; 15(4):1945-1953.

19. Brasil. Ministério da Saúde. Política Nacional de Atenção Básica. Brasil, 2011.

20. Paim JS. Reforma sanitária brasileira: contribuição para a compreensão e crítica. Salvador: Edufba; Rio de Janeiro: Fiocruz; 2008.

21. Lavalle AG, Vera EI. Representación y participación em la crítica democrática. Desacatos 49, septiembre-diciembre 2015; 10-27.

22. Sumar N, Fausto MCR. Atenção Primária à Saúde: a construção de um conceito ampliado. J Manag Prim Health Care 2014; 5(2):202-212.

23. Heimann LS, Ibanhes LC, Boaretto RC, Castro IEN, Telesi Júnior E, et al. Atenção primária em saúde: um estudo multidimensional sobre os desafios e potencialidades na Região Metropolitana de São Paulo (SP, Brasil). Ciênc. saúde colet. 2011; 16(6):2877-2887.

24. Lei $n^{\circ} 9.637$ de 15 de Maio de 1998. Dispõe sobre a qualificação de entidades como organizações sociais, a criação do Programa Nacional de Publicização, a extinção dos órgãos e entidades que menciona e a absorção de suas atividades por organizações sociais, e dá outras providências. Diário Oficial da União 1998; 18 mai.

25. Lei $n^{\circ} 9.790$ de 23 de Março de 1999. Dispõe sobre a qualificação de pessoas jurídicas de direito privado, sem fins lucrativos, como Organizações da Sociedade Civil de Interesse Público, institui e disciplina o Termo de Parceria, e dá outras providências. Diário Oficial da União 1999; 24 mar.

26. Barboza R, Morais, MSL. Avanços e impasses da Estratégia Saúde da Família: a percepção dos gestores e gerentes da Região Metropolitana da Baixada Santista. BIS, bol. Inst. Saúde 2014; 15(2):34-42.

27. Starfield B. Atenção Primária: equilíbrio entre necessidades de saúde, serviços e tecnologia. Brasília: UNESCO; Ministério da Saúde; 2004.

28. Mendes EV. 25 anos do Sistema Único de Saúde: resultados e desafios. Estud. av. 2013; 34 
JMPHC

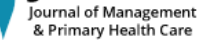

ISSN 2179-6750

27(78):27-34.
JMPHC. Journal of Management and Primary Health Care. www.jmphc.com.br J Manag Prim Heal Care. 2015; 6(1):18-35. 